\title{
Comparison of three isolation systems for the culture of mycobacteria from respiratory and non-respiratory samples
}

\author{
G Harris, A Rayner, J Blair, B Watt
}

\begin{abstract}
Aims-To compare the recovery of mycobacteria from clinical samples using the $M B / B a c T$ rapid culture system with that obtained using egg medium or the Bactec radiometric method.

Methods-The three methods were compared using 681 clinical samples (462 respiratory and 219 non-respiratory samples) and eight external quality control strains. Culture media were incubated at $35-37^{\circ} \mathrm{C}$ for six weeks in the $\mathrm{MB} / \mathrm{BacT}$ system and for 12 weeks in the Bactec system and on egg medium. Solid media were examined macroscopically once a week and the Bactec vials were read six times in the first two weeks, and then weekly for the next 10 weeks (a growth index $>50$ indicated a positive vial). The $\mathrm{MB} / \mathrm{BacT}$ system positive vials were unloaded from the machine as soon as possible after detection. Confirmation of growth for all systems was by Ziehl-Neelson stained smears. Isolates were identified by a combination of phenotypic and molecular methods.
\end{abstract}

Results-Of the 681 clinical samples, 59 $(8.7 \%)$ were positive on culture, including 23 strains of Mycobacterium tuberculosis. None of the three systems recovered all of the isolates, but each recovered mycobacteria not detected by either of the other two systems. After six weeks incubation, isolation rates were $87 \%, 78 \%$, and $90 \%$, and mean times to detection were 13,19 , and nine days for the $M B / B a c T$, egg medium, and Bactec systems, respectively. Although the MB/BacT system was slightly slower than the Bactec system, the biomass was greater, allowing earlier use of molecular probes and earlier inoculation of susceptibility tests.

Conclusions-The MB/BacT system provides comparable performance to the Bactec radiometric system, without the problems of disposal of radioactive waste. Optimal recovery is obtained when culture on egg medium is used in conjunction with a rapid culture system.

(F Clin Pathol 2000;53:615-618)

Keywords: mycobacteria; rapid culture; solid media

The increased interest in mycobacterial infections in humans has led to a corresponding demand for more rapid laboratory diagnosis than can be achieved by conventional culture procedures. This has recently been formalised by the Centers for Disease Control and Prevention (CDC; Atlanta, Georgia, USA) as a requirement for a turn round time for isolation and identification of Mycobacterium tuberculosis of 14-21 days. ${ }^{1}$ Such times can be achieved by detection of amplified nucleic acid sequences in clinical samples, or by rapid liquid culture systems. At present, molecular technologies are expensive, and commercial molecular systems might have problems of sensitivity and specificity, ${ }^{2}$ so rapid liquid culture systems offer an important alternative.

In our laboratory, we have used the Bactec 460 radiometric system for some years for rapid culture of mycobacteria. More recently, we have installed an automated nonradiometric system (MB/BacT; Organon Teknika, Cambridge, UK); our paper compares the performance of the two systems with each other and with egg medium, in recovering mycobacteria of clinical interest from a range of respiratory and non-respiratory samples.

Materials and methods

SAMPLES

A total of 681 clinical samples and eight NEQAS quality control samples received at the Scottish mycobacteria reference laboratory (SMRL) were used in the evaluation. The clinical samples included 462 respiratory samples (sputum and bronchial lavage samples) and 219 non-respiratory samples (urine, faeces, tissues, cerebrospinal fluid, other fluids and aspirates, and wound swabs). Before culture, all clinical samples were examined by direct microscopy (auramine-phenol (AP) stain), with all positive smears confirmed by Ziehl-Neelson (ZN) staining. ${ }^{3}$

\section{CULTURE PROCEDURES}

Samples were treated using either $4 \%$ sodium hydroxide or $3 \%$ oxalic acid (depending on sample type) followed by centrifugation at $2000 \times g$ for 20 minutes. Each deposit was then neutralised using either $14 \%$ dipotassium orthophosphate or $2 \%$ sodium hydroxide as appropriate (phenol red as indicator). After neutralisation, each deposit was made up to $2 \mathrm{ml}$ using sterile phosphate buffer ( $\mathrm{pH}$ 6.8), and $0.5 \mathrm{ml}$ aliquots were inoculated into an $\mathrm{MB}$ process bottle, a Bactec $12 \mathrm{~B}$ vial, and on to one each of a glycerol and sodium pyruvate egg slope. ${ }^{3}$ Each of the liquid media contained an antibiotic "cocktail" consisting of polymixin, amphotericin B, naladixic acid, trimethoprim,
Accepted for publication 20 January 2000 
Table 1 Comparative recovery of the 67 mycobacterial isolates by culture method

\begin{tabular}{lllll}
\hline Culture method & $\begin{array}{l}\text { Total incubation } \\
\text { period (weeks) }\end{array}$ & $\begin{array}{l}\text { No. (\%) of positive } \\
\text { isolates in stated system }\end{array}$ & $\begin{array}{l}\text { Mean time to } \\
\text { detection (days) }\end{array}$ & $\begin{array}{l}\text { Range } \\
\text { (days) }\end{array}$ \\
\hline MB/BacT & 6 & $58(87)$ & 13 & $3-33$ \\
& 8 & $\mathrm{ND}$ & $\mathrm{ND}$ & $\mathrm{ND}$ \\
Bactec & 12 & $\mathrm{ND}$ & $\mathrm{ND}$ & $\mathrm{ND}$ \\
& 6 & $60(90)$ & 9 & $2-35$ \\
Egg medium & 8 & $60(90)$ & 9 & $2-35$ \\
& 6 & $61(91)$ & 10 & $2-62$ \\
& 12 & $52(78)$ & 19 & $7-42$ \\
& 12 & $55(82)$ & 21 & $7-56$ \\
\end{tabular}

The MB/BacT system protocol requires incubation for six weeks only.

ND, not done.

Table 2 Recovery of mycobacteria from respiratory samples at six weeks by culture method

\begin{tabular}{llll}
\hline \multirow{2}{*}{\begin{tabular}{l} 
Species (no. isolated) \\
\cline { 2 - 4 }
\end{tabular}} & \multicolumn{4}{l}{ Percentage of positive isolates (time to detection in days (range)) } \\
\cline { 2 - 4 } MB/BacT & Egg medium & Bactec 460 \\
\hline All mycobacteria (44) & $86(12.8(3-30))$ & $66(18.6(14-42))$ & $91(9.4(2-35))$ \\
M tuberculosis (18) & $94(15.3(7-30))$ & $78(19.1(14-28))$ & $100(11.7(4-35))$ \\
$M$ mavimoense (15) & $80(14.3(3-22))$ & $47(29.7(21-42))$ & $73(10.2(3-21))$ \\
$M$ fortuitum (7) & $75(8.7(6-22))$ & $25(14.0)$ & $100(7.0(3-21))$ \\
\hline
\end{tabular}

and azlocillin ("PANTA", Becton Dickinson, Cowley, Oxford, UK; "MAS", Organon Teknika).

INCUBATION

All culture media were incubated at $35-37^{\circ} \mathrm{C}$. $\mathrm{MB} / \mathrm{Bac} \mathrm{T}$ process bottles were loaded into the $\mathrm{MB} / \mathrm{Bac} \mathrm{T}$ cabinet on the day of inoculation; negative bottles were unloaded after six weeks incubation; positive bottles were unloaded as soon as possible after detection by the machine. Both the Bactec $12 \mathrm{~B}$ vials and the solid media were incubated for 12 weeks.

Solid media were examined macroscopically once a week and Bactec $12 \mathrm{~B}$ vials were read six times in the first two weeks and then weekly for the next 10 weeks. A growth index $>50$ was used as an indicator of a positive Bactec vial. Confirmation of mycobacterial growth for all culture systems was by $\mathrm{ZN}$ stained smears. Purity was checked by inoculation on to Columbia agar (Oxoid, Basingstoke, Hampshire, UK) containing 5\% horse blood (blood agar), incubated aerobically overnight at $37^{\circ} \mathrm{C}$, and then examined for growth.

\section{IDENTIFICATION}

All isolates were identified to species level, using a combination of conventional and molecular methods. Briefly, all $M$ tuberculosis isolates were confirmed by colonial morphology and the production of niacin. Mycobacteria other than tuberculosis (MOTT) isolates were identified either conventionally (using temperature preference, oxygen preference, ability to hydrolyse Tween, and pigment production ${ }^{4}$ ) or by "Accuprobe" (Gene Probe, San Diego, California, USA) molecular probes specific for $M$ avium complex. In addition, for the purpose of our study, molecular identification using either "Accuprobe" $M$ tuberculosis or $M$ avium complex probes was also performed directly on 39 positive $\mathrm{MB} / \mathrm{BacT}$ process bottles. Inoculum for these latter tests was $0.1 \mathrm{ml}$ from each positive $\mathrm{MB} / \mathrm{BacT}$ process bottle.
CALCULATION OF TIME TO SUSCEPTIBILITY INOCULATION

In our laboratory, inoculated Bactec $12 \mathrm{~B}$ vials are used to seed susceptibility vials when the growth index reaches 700 or greater. Initially, Bactec 12B vials were inoculated with $0.2 \mathrm{ml}$ from positive $\mathrm{MB} / \mathrm{Bac} \mathrm{T}$ process bottles and then incubated until the appropriate growth index was achieved for inoculation of a Bactec susceptibility test. The length of this incubation period was recorded and added to the time to isolation, to give "time to susceptibility inoculation" (TSI). However it became apparent that Bactec susceptibility tests could be inoculated directly from positive $\mathrm{MB} / \mathrm{BacT}$ process bottles using an appropriate dilution. Therefore, for the MB/BacT system, the TSI was taken purely as the length of incubation until the bottle was determined positive. For the Bactec system, the number of days incubation needed to achieve a growth index of 700 was recorded, and this was taken as the TSI.

CONTAMINATION RATES

Contamination in the liquid culture systems was detected by subculture on to blood agar. If an $\mathrm{MB} / \mathrm{BacT}$ process bottle or a Bactec $12 \mathrm{~B}$ vial was $\mathrm{ZN}$ negative and showed nonmycobacterial growth on blood agar it was recorded as contaminated and discarded. Solid medium contamination was determined by $\mathrm{ZN}$ smear and the ability of the slope to support mycobacterial growth. If more than half of the surface of a given egg slope showed growth that was $\mathrm{ZN}$ negative on microscopy then that slope was considered to be contaminated and discarded. For the purpose of our study, solid media were only recorded as contaminated if both the glycerol and pyruvate slopes were overgrown with contaminants. No contaminated slopes showed any concomitant mycobacterial growth.

\section{Results}

Of the 681 clinical samples, $59(8.7 \%)$ were positive on culture, yielding the following organisms: M tuberculosis (23), M malmoense (15), $M$ avium complex (13), $M$ fortuitum (seven), and $M$ bovis (one). Of the 59 isolates, $44(75 \%)$ were from respiratory samples, 18 of which yielded $M$ tuberculosis on culture. Fifty two $(88 \%)$ of the culture positive samples were smear positive.

None of the systems cultured $100 \%$ of the clinical isolates, but each of the systems isolated mycobacteria that were not detected by either of the other systems. All of the NEQAS quality control samples grew in all three culture systems, yielding seven isolates of $M$ tuberculosis and one of M kansasii. Two clinical samples were lost through contamination in all three culture systems. Table 1 summarises the results for all culture systems at six weeks and for the Bactec and egg systems for eight and 12 weeks. At six weeks, the isolation rates for the $\mathrm{MB} / \mathrm{BacT}$, egg media, and Bactec were $87 \%, 78 \%$, and $90 \%$, respectively, with mean times to detection of 13, 19, and nine days. By the end of 12 weeks incubation, the rates for egg media and Bactec had changed 
Table 3 Recovery of mycobacteria from non-respiratory samples at six weeks by culture method

\begin{tabular}{|c|c|c|c|}
\hline \multirow[b]{2}{*}{ Species (no. isolated) } & \multicolumn{3}{|c|}{ Percentage of positive isolates (time to detection in days (range)) } \\
\hline & $M B / B a c T$ & Egg medium & Bactec 460 \\
\hline All mycobacteria (15) & $80(15.3(8-33))$ & $87(20.4(14-35))$ & $80(8.6(3-13))$ \\
\hline$M$ tuberculosis (5) & $100(17.4(15-21))$ & $100(18.2(14-21))$ & $100(11.2(10-13))$ \\
\hline$M$ bovis (1) & 0 & 100 & 100 \\
\hline & & 14.0 & \\
\hline$M$ avium complex (9) & $78(13.7(8-33))$ & $78(22.9(14-35))$ & $67(6.7(3-8))$ \\
\hline
\end{tabular}

Table 4 Time to susceptibility test inoculation *

\begin{tabular}{lll}
\hline & \multicolumn{2}{l}{ Time to inoculation (days) } \\
\cline { 2 - 3 } Species (no. tested) & MB/BacT & Bactec \\
\hline All mycobacteria (27) & 12.3 & 12.0 \\
M tuberculosis (17) & 12.8 & 11.5 \\
$M$ malmoense (5) & 14.0 & 18.4 \\
$M$ avium complex (5) & 9.2 & 7.0 \\
\hline
\end{tabular}

^See Materials and methods for definition.

to $85 \%$ and $91 \%$, respectively. Tables 2 and 3 show the results separately for respiratory and non-respiratory samples. At six weeks, there were nine "false negative" samples on the $\mathrm{MB} / \mathrm{BacT}$ system, 15 on egg medium, and seven by Bactec. The number of false negative samples reduced with further incubation for the egg and Bactec systems, to 10 and six, respectively, at 12 weeks. If false negatives as a result of contamination are excluded, the false negative rates at six weeks are five, 10 , and four for $\mathrm{MB} / \mathrm{BacT}$, egg, and Bactec media, respectively. Table 4 shows the mean TSI for 27 isolates of mycobacteria from the $\mathrm{MB} / \mathrm{BacT}$ and Bactec culture systems. Twelve days was the mean TSI for the 27 isolates by both rapid systems. The $M$ tuberculosis isolates (17) averaged 12 days by Bactec and 13 days by $\mathrm{MB} / \mathrm{BacT}$. The five $M$ avium complex isolates took two days longer by MB/BacT than Bactec but the five $M$ malmoense isolates were four days faster using $\mathrm{MB} / \mathrm{BacT}$ as the primary isolation system.

Times to detection and percentage recovery of the 59 clinical isolates were compared for different "degrees of positivity" of smears for microscopy (table 5). As might be expected, times to detection were in general lower and percentage recovery higher for strongly smear positive samples than for smear negative ones, especially for the $\mathrm{MB} / \mathrm{BacT}$ system, but there was no close correlation.

The overall contamination rates were $6.7 \%$, $5.1 \%$, and $1.7 \%$ for the $\mathrm{MB} / \mathrm{BacT}$, egg medium, and Bactec systems, respectively. The rates for the $\mathrm{MB} / \mathrm{BacT}$ system and for egg medium were higher than our normal values $(2-5 \%)$, probably as a result of increased han-

Table 5 Correlation of time to detection and recovery with sample microscopy for 59 clinical isolates of mycobacteria

\begin{tabular}{lllll}
\hline \multirow{2}{*}{ Microscopy } & \multirow{2}{*}{$\begin{array}{l}\text { No. of } \\
\text { isolates }\end{array}$} & \multicolumn{4}{l}{ Mean days to detection (\% recovery) with system } \\
\cline { 3 - 5 } & $M B / B a c T$ & Solid medium & Bactec \\
\hline Negative & 7 & $16.0(71.4)$ & $27.8(71.4)$ & $7.7(85.7)$ \\
+ & 20 & $18.8(80.0)$ & $29.9(80.0)$ & $13.7(85.0)$ \\
++ & 10 & $8.8(80.0)$ & $26.8(90.0)$ & $12.8(90.0)$ \\
+++ & 22 & $10.4(90.9)$ & $17.5(86.4)$ & $6.8(90.9)$ \\
\hline
\end{tabular}

+ Weakly positive; ++ positive; +++ strongly positive. dling of samples before inoculation of the culture systems and unfamiliarity with the new $\mathrm{MB} /$ BacT system. Training of a new member of staff also caused an increase in contamination rates over a one week period; if this week is excluded, the contamination rates are $5.5 \%$, $4.9 \%$, and $1.8 \%$, respectively. Ninety five per cent of the $\mathrm{MB} / \mathrm{BacT}$ contaminants were Gram positive cocci.

\section{Discussion}

The culture of mycobacteria from clinical samples on solid medium has always been a labour intensive and slow procedure because the sample requires treatment before it can be cultured, and mycobacteria may take several weeks to grow, (although detection of visible colonies on solid medium is quicker with experienced staff). Therefore, there has been much interest in more rapid and automated systems for the recovery of mycobacteria from clinical samples. The first of these was the Bactec 460TB radiometric system, which was much faster than solid medium, ${ }^{56}$ but is only semiautomated (still requiring manual loading and unloading and result transcription). Disposal of radioactive waste is another drawback of the system.

The MB/BacT system is one of the next generation of mycobacteria detection systems, named CAMLiC (continuous automated mycobacteria liquid culture system) by Magee et $a l .^{7}$ It is fully automated and non-radiometric. Our study showed that the system gave comparable isolation rates; times to detection were slightly longer than for the Bactec system, but this was counteracted by the fact that isolates detected in the latter system required further incubation before there was sufficient biomass to allow susceptibility tests and molecular identification tests to be performed, whereas a positive $\mathrm{MB} / \mathrm{BacT}$ process bottle can be used immediately for further susceptibility and molecular identification tests. We also found that identification using molecular probes was easier to perform on $\mathrm{MB} / \mathrm{Bac} \mathrm{T}$ process bottles because no centrifugation step was required (unlike Bactec bottles). Our contamination rate for the $\mathrm{MB} / \mathrm{BacT}$ system was comparable to that obtained by Magee et al.

The development of rapid culture systems raises the question of whether these should be adopted by laboratories instead of, or as an adjunct to, conventional solid media. Our present study shows clearly that if culture on egg medium is combined with $\mathrm{MB} / \mathrm{BacT}$ or the Bactec systems, the overall recovery rate at six weeks is 62 of $65(95.4 \%)$ for both combinations, respectively. (Further incubation of the Bactec and solid medium for a total of 12 weeks gave a recovery rate of 66 of 67 or $98.5 \%$.) No single system isolated all of the clinical strains. We intend to continue using egg medium in combination with rapid culture systems for the foreseeable future.

There have been several published studies on the $\mathrm{MB} / \mathrm{BacT}$ system. Garcia and colleagues ${ }^{8}$ compared the $\mathrm{MB} / \mathrm{BacT}$ system with culture on egg medium for isolation of mycobacteria (predominantly $M$ tuberculosis) from a variety 
of clinical samples. Of the 93 strains isolated, $88(94.6 \%)$ grew in the $\mathrm{MB} / \mathrm{BacT}$ system compared with 66 (71\%) on egg medium; mean times to detection were 16.5 and 22.7 days, respectively. The study of Magee et al involved a total of 2800 sputum samples that yielded 191 strains of mycobacteria, with a mean time to detection of 13.4 days. $^{\text {? }}$

Palacios et al, in their study of 5208 samples, showed that the $\mathrm{MB} / \mathrm{BacT}$ recovered $96.1 \%$ of the 257 isolates of $M$ tuberuclosis compared with $72.7 \%$ on egg medium ${ }^{9}$; times to detection were 11.5 and 16.5 days, respectively.

Two studies compared the MB/BacT system with the Bactec 460 radiometric system. Benjamin et al studied a total of 488 specimens, which yielded $39(89 \%)$ of 44 isolates in the $\mathrm{MB} / \mathrm{BacT}$ system and $40(91 \%)$ with the Bactec 460 system. ${ }^{10}$ Time to detection for the two systems was broadly comparable, although slightly longer for the $\mathrm{MB} / \mathrm{Bac} \mathrm{T}$ system. The authors concluded that the $\mathrm{MB} / \mathrm{BacT}$ system was: "an acceptable alternative for use in the diagnostic laboratory".

Apart from our study, there is only one other published study comparing the $\mathrm{MB} / \mathrm{Bac} \mathrm{T}$ system with both the Bactec 460 system and solid medium. Rohner and colleagues ${ }^{11}$ obtained an isolation rate of $86.3 \%$ with the $\mathrm{MB} / \mathrm{BacT}$ system, compared with $91.8 \%$ for the Bactec system, and $79.5 \%$ for egg medium. The mean (SD) time to detection of positive growth was 17.5 (6.4) days for the $\mathrm{MB} / \mathrm{BacT}$ and 14.3 (8.2) days for the Bactec system, compared with 13 days and nine days, respectively, in our study.

In many laboratories it is good practice to incubate cultures on egg medium for up to 12 weeks, possibly longer in certain circumstances. In addition, the facility to incubate at different temperatures is important; for example $M$ marinum is recovered optimally from clinical samples at $30^{\circ} \mathrm{C}$. Therefore, the inability of the $\mathrm{MB} / \mathrm{BacT}$ system to allow incubation at temperatures other than $37^{\circ} \mathrm{C}$ is a disadvantage.

There are other rapid culture systems under development and these might prove useful alternatives to present systems; we shall evalu- ate them in due course. At present, the Bactec radiometric system is the only one that has been validated for susceptibility testing; the eventual development of non-radiometric systems that can be used for both isolation and susceptibility testing on a "seamless" basis for a given test strain will be a welcome development.

Rapid culture systems, or CAMLiCs, ${ }^{7}$ will continue to be an important component in the laboratory diagnosis of mycobacterial infections for the foreseeable future. The $\mathrm{MB} / \mathrm{BacT}$ automated system provides comparable performance to the Bactec semiautomated radiometric system, without the problems associated with the disposal of radioactive waste.

We are grateful to Organon Teknika for financial support towards the cost of our study and to Miss Lorraine Gray for expert secretarial support.

1 Styrt BA, Shinnick TM, Rudderhof JC, et al. Turnaround times for mycobacterial cultures. 7 Clin Microbiol 1997;35:1041-2.

2 Vuorinen P, Miettinen A, Vuento R, et al. Direct detection of Mycobacterium tuberculosis complex in respiratory specimens by Gene-probe amplified Mycobacterium tuberculosis direct test and Roche amplicor Mycobacterium sis direct test and Roche amplicor Mycobacte

3 Watt B, Rayner A, Harris G. Modern methods in mycobacteriology. Reviews in Medical Microbiology 1993;4:97-105.

4 Marks J. A system for the examination of tubercle bacilli and other mycobacteria. Tubercle 1976;57:207-25.

5 Roberts GD, Goodman NL, Heifets L, et al. Evaluation of the Bactec radiometric method for recovery of mycobacteria and drug susceptibility testing of Mycobacterium tuberculosis from acid-fast smear-positive specimens. $\mathcal{F}$ Clin Microbiol 1983;18:689-96.

6 Rayner A, Harris G, Croughan M, et al. Use of a radiometric technique for rapid sensitivity testing of mycobacteria in Scotland; the first year's experience. Scott Med F 1991;35: $142-4$

7 Magee JG, Freeman R, Barnett A. Enhanced speed and sensitivity in the cultural diagnosis of pulmonary tubercuosis with a continuous automated mycobacterial liquid culture (CAMLiC) system. 7 Med Microbiol 1998;47:547 53.

8 Garcia FG, Angulo GP, Garcia FG, et al. Evaluation of the $\mathrm{MB} / \mathrm{BacT}$ automated mycobacteria culture system versus culture on Lowenstein medium. Clinical Microbiology and Infection 1998;4;339-43.

9 Palacios JJ, Ferro J, Palma NR. Fully automated liquid culture system compared with Lowenstein-Jensen solid medium for rapid recovery of mycobacteria from clinical samples. Eur F Clin Microbiol Infect Dis 1999;18:265-73.

0 Benjamin WH, Jr, Waites KB, Beverly A, et al. Compariso of the $\mathrm{MB} / \mathrm{BacT}$ system with a revised antibiotic supplement kit to the Bactec 460 system for detection of mycobacteria in clinical specimens. $f$ Clin Microbiol 1998;36:3234-8

11 Rohner P, Ninet B, Metral C, et al. Evaluation of the $\mathrm{MB} / \mathrm{BacT}$ system and comparison to the Bactec 460 system and solid media for the isolation of mycobacteria from clinical specimens. f Clin Microbiol 1997;35:3127-31. 